\title{
Unusual muscle pathology in McLeod syndrome
}

\author{
M H Barnett, F Yang, H Iland, J D Pollard
}

\begin{abstract}
Muscle pathology in McLeod syndrome is usually mild; patchy necrotic or regenerating fibres, occasional internal nuclei, and the absence of an inflammatory cell infiltrate are the usual findings. We report on a 29 year old man presenting with chronic fatiguability and excessive sweating in whom an open quadriceps muscle biopsy demonstrated grouped necrotic fibres accompanied by striking patchy mononuclear cell infiltrates. The diagnosis of McLeod syndrome was made on the basis of red blood cell acanthocytosis, raised serum creatine kinase, and weak expression of Kell blood group antigens. The quadriceps muscle infiltrate consisted principally of histologically typical macrophages. These cells had prominent nucleoli, displayed numerous mitoses, and were strongly CD68+. A small population of typical CD3+, CD43+ lymphocytes was also present. In addition, a small population of large atypical CD3+ cells was noted. Immunoperoxidase stains for CD20, CD30, CD79a, and CD56 were negative. Immunocytochemical studies for the common muscular dystrophies were normal. The muscle biopsy findings highlight a potential for confusion of this condition with idiopathic polymyositis. The expanding range of muscle pathology reported in McLeod syndrome, to which this case adds, may reflect variable involvement of the $X K$ gene on chromosome Xp21, or of the adjacent loci of Duchenne muscular dystrophy and chronic granulomatous disease.
\end{abstract}

(F Neurol Neurosurg Psychiatry 2000;69:655-657)

Keywords: McLeod syndrome; myopathy; muscle biopsy

McLeod syndrome is one of several neuromuscular disorders associated with acanthocytosis. ${ }^{1}$ It is characterised by myopathy, choreiform movements, raised creatine kinase, and persistent low grade haemolysis. Peripheral neuropathy, cardiomyopathy, and atrophy of the caudate nuclei on MRI are less often seen. ${ }^{1-3}$ The diagnosis is suggested by the presence of acanthocytosis in the appropriate clinical setting, and confirmed by the depressed expression of the Kell blood group antigens, and the absence of the $\mathrm{Kx}$ antigen. ${ }^{4}$ The responsible gene, $X K$, is located on chromosome Xp21, between the loci of chronic granulomatous disease and Duchenne muscular dystrophy ${ }^{5}$; rarely, these disorders may occur concurrently. ${ }^{6}$ The muscle biopsy findings are variable, but usually subtle. ${ }^{2}$ We report on a patient with the McLeod phenotype and striking atypical mononuclear cell infiltrates on muscle biopsy.

\section{Case report}

A 29 year old carpenter presented with a 5 year history of persistent fatigue and excessive sweating on minimal exertion, initially attributed to a febrile illness diagnosed as infectious mononucleosis. There were no classic night sweats or weight loss, nor any myalgias or definite weakness. His symptoms were exacerbated by a diarrhoeal illness while in Egypt 18 months after onset, at which point he sought medical attention. Creatine kinase (CK) concentrations at the time were increased to 2000-3000 U/1, and a subsequent needle EMG was consistent with a patchy myopathic process. Persistently abnormal CK concentrations led to an open muscle biopsy of the right quadriceps, which was reported as consistent with a necrotising myopathy. Patchy cellular infiltrates, not typical of polymyositis, were noted at the time. The patient was nevertheless immunosuppressed with high dose prednisolone and subsequently azathioprine and oral cyclophosphamide without appreciable benefit. He was referred to our institution for review.

There was no known family history of neuromuscular disease. General examination was unremarkable, and specifically there was no rash, synovitis, lymphadenopathy, or hepatosplenomegaly. There was no muscle tenderness, and no focal neurological findings. The reflexes were present and symmetric, and there were no sensory abnormalities.

Biochemical screening disclosed a raised CK of $790 \mathrm{U} / 1(0-250 \mathrm{U} / 1)$, and a mildly raised serum bilirubin and LDH. Serum haptoglobins were depressed. The reticulocyte count was not increased and there was no myoglobin in the urine. A mild thrombocytopenia of $137 \times 10^{9} / 1$ platelets was present on an otherwise unremarkable full blood count. The blood film, however, disclosed marked acanthocytosis (about $35 \%$ of erythrocytes) and occasional spherocytes. Serum ANA, ENA, RF, ANCA, $\mathrm{HIV}$, and quantitative immunoglobulins were 


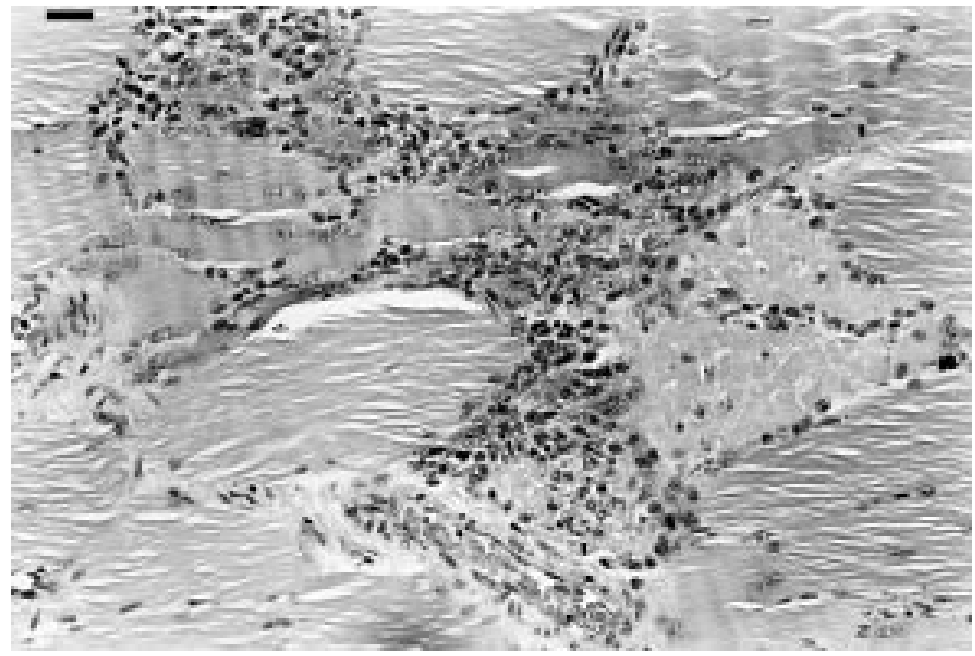

Left quadriceps muscle biopsy; haematoxylin and eosin. Necrotic fibres with histiocytic cell infiltrate. Bar=1 $\mu \mathrm{m}$. have, to our knowledge, not been previously reported in McLeod syndrome. They highlight a potential for confusion of this condition with idiopathic polymyositis, in which patchy inflammatory infiltrates consist predominantly of CD8+ T cells. Although macrophages may be seen in association with degenerating muscle fibres various myopathies including the autoimmune myositides, the conspicuous infiltrates and paucity of associated lymphocytes are unusual. Our patient received potentially harmful therapy with prednisone, azathioprine, and oral cyclophosphamide before the diagnosis became apparent. Recognition of the wide phenotypic range of McLeod syndrome, both clinical and histopathological, could help avert such a scenario. Muscle biopsy in most patients exhibits myopathic changes including scattered necrotic and regenerating fibres, internal nuclei, and the absence of inflammatory cell infiltration. ${ }^{28}$ Kawakami et al reported more advanced myopathic changes reminiscent of the muscular dystrophies in a patient with McLeod syndrome; however, again no inflammatory cell infiltrates were seen. ${ }^{9}$ Similarly the clinical symptoms range from asymptomatic to overt weakness, with or without associated cardiomyopathy, choreoathetosis, or peripheral neuropathy. ${ }^{12}$ Atrophy of the caudate nuclei on MRI of the brain has been reported, but was not evident in our patient.

The underlying genetic heterogeneity of McLeod syndrome may account for the described clinical and pathological diversity. ${ }^{51011}$ The proximity of the responsible gene, $X K$, to the loci of CGD and DMD accounts for the rare concurrence of these diseases, presumably due to large deletions of the $\mathrm{X}$ chromosome. ${ }^{67}$ Preliminary analysis of our patient's XK gene by direct sequencing has established the presence of a novel missense point mutation. ${ }^{12}$

Examination of the peripheral blood film in this patient prompted further investigation and led to the diagnosis of McLeod syndrome. Reviewing red cell morphology for the presence of acanthocytosis is a sensitive and inexpensive screening test for the McLeod syndrome, and should be performed for any patient who carries a diagnosis of inflammatory muscle disease with either atypical biopsy findings or poor response to immunosuppressive therapy.

We thank Mrs M Nelson for performing flow cytometry for Kell antigen expression.

Kell antigen expression was evaluated by flow cytometry with polyclonal anti-Kell antibodies. Expression of both members of the antithetical pair Kell (K1) and Cellano (K2) was reduced, consistent with the McLeod syndrome. Anti-Kx antibodies for definitive demonstration of absent $\mathrm{Kx}$ expression were not available.

\section{Discussion}

The McLeod phenotype in this patient was confirmed by the weak expression of Kell blood group antigens. The striking cellular infiltrates demonstrated in this man's muscle biopsy
1 Hardie RJ, Pullon HWH, Harding AE, et al. Neuroacanhocytosis: a clinical, haematological and pathological study of 19 cases. Brain 1991;114:13-49.

2 Swash M, Schwartz MS, Carter ND, et al. Benign $x$ linked myopathy with acanthocytes (McLeod syndrome). Brain 1983;106:717-33. McLeod syndrome. Neurology 1994;44:117-20.

4 Marsh WL, Redman CM. Recent developments in the Kell blood group system. Transfusion Med Rev 1987;1:4-20.

5 Ho M, Chelly J, Carter N, et al. Isolation of the gene for McLeod syndrome that encodes a novel membrane transport protein. Cell 1994;77:869-80.

6 Francke U, Ochs HD, De Martinville B, et al. Minor Xp21 chromosome deletion in a male associated with expression of Duchenne muscular dystrophy, chronic granulomatous disease, retinitis pigmentosa, and McLeod syndrome. Am $\mathcal{F}$ Hum Genet 1985;37:250-67.
3 Danek A, Uttner I, Vogl T, et al. Cerebral involvement in 
7 Frey D, Mächler M, Seger R, et al. Gene deletion in a patient with chronic granulomatous disease and McLeod syndrome: fine mapping of the XK gene locus. Blood 1988; $71: 252-5$

8 Zyskowski LP, Bunch TW, Hoagland HC, et al. McLeod syndrome (hemolysis, acanthocytosis, and increased creatine kinase): potential confusion with polymyositis. Arthritis Rheum 1983;26:806-8.

9 Kawakami T, Takiyama Y, Sakoe K, et al. A case of Mcleod syndrome with unusually severe myopathy. $\mathrm{f}$ Neurol Sci 1999;166:36-9.
10 Hanaoka N, Yoshida K, Nakamura A, et al. A novel frameshift mutation in the McLeod syndrome gene in a frameshift mutation in the McLeod synd

11 Ho MF, Chalmers RM, Davis MB, et al. A novel point mutation in the McLeod syndrome gene in neuroacanthocytosis. Ann Neurol 1996;39:672-5.

12 Iland HJ, Supple SG, Barnett MH, et al. A novel XK gene mutation in a patient with McLeod syndrome and unusual muscle pathology. Proceedings of Congress of The Haematology Society of Australia and New Zealand, 2000. Perth, Australia: HSANZ, July 2000. 